\title{
How large must an iridotomy be?
}

\author{
B W Fleck
}

\begin{abstract}
Four cases of acute angle closure glaucoma in eyes with a small but patent Nd-YAG laser iridotomy are presented, and similar cases in the literature are reviewed. Theoretically a 15 $\mu \mathrm{m}$ diameter iridotomy should be large enough to prevent angle closure glaucoma due to pupil block. Mechanisms by which larger iridotomies fail to prevent angle closure glaucoma, and the role of provocation tests following iridotomy, are discussed. An iridotomy should be at least $150-200 \mu \mathrm{m}$ in diameter if acute angle closure glaucoma is to be reliably prevented.
\end{abstract}

Various forms of iris surgery have been used to treat angle closure glaucoma since von Graefe's original description of the broad iridectomy in 1857.' Surgical iridotomy was introduced by Curran in $1920 . .^{2-4}$ Peripheral iridectomy was described by Chandler in 1952. Argon laser iridotomy, ${ }^{6}$ and more recently Nd-YAG laser iridotomy, ' have already largely superseded surgical iridectomy. While laser surgery has many advantages over conventional surgery, it must not be forgotten that laser iridotomy represents a return to the use of smaller iris openings than those obtained by surgical peripheral iridectomy. ${ }^{8}$ Gifford noted in 1921 that very small surgical iridotomies were not as effective in controlling intraocular pressure as larger iridotomies. ${ }^{4}$

Reports are now emerging of acute angle closure glaucoma developing in the presence of a small but patent $\operatorname{argon}^{910}$ or Nd-YAG ${ }^{11}{ }^{12}$ laser iridotomy. While cases of acute angle closure glaucoma following surgical peripheral iridectomy have generally been attributed to plateau iris syndrome, ${ }^{13-15}$ not all cases of acute angle closure glaucoma following a small laser iridotomy can be placed in this category. ${ }^{9-12}$ We have treated four patients who developed acute angle closure glaucoma despite the presence of a patent Nd-YAG laser iridotomy.

The mechanism of failure of very small iridotomies may be related to obstruction of aqueous flow at very small iridotomy size. Below a critical surface area significant iris bombé may occur at normal aqueous flow rates. When iris bombé is sufficient to cause iridocorneal contact throughout a critical extent of the angle, beginning superiorly, acute angle closure glaucoma results. ${ }^{1617}$ The minimum 'safe' size of iridotomy has not been defined, but an approximate value may be calculated from a simple mathematical model.

Material and methods

We are undertaking a prospective randomised comparison of Nd-YAG laser iridotomy and surgical peripheral iridectomy in the treatment of acute angle closure glaucoma. The cases presented belong to the laser treated group in this study.

A Zeiss Visulas Nd-YAG laser and Zeiss anterior segment YAG contact lens were used in all treatments. Patients were given acetazolamide $500 \mathrm{mg}$ orally one hour before treatment, and one pilocarpine eyedrop $2 \% 30$ minutes before treatment.

A treatment site was chosen in the superior iris between 10 and 2 o'clock, approximately twothirds of the distance from the pupil margin to the base of the iris. An iris crypt was used, when present. A single 5-10 mJ pulse was delivered to the treatment site. If this pulse did not penetrate the iris, further single pulses were delivered to the same site until penetration was achieved. A gush of aqueous fluid from the posterior chamber could be seen at the moment of penetration. Patency was assessed by direct observation of the posterior chamber or anterior lens capsule.

The size of iridotomies was measured by comparison with the $0.2 \mathrm{~mm}$ spot produced by a Haag-Streit slit-lamp focused on the iris plane.

\section{Results: case reports}

CASE I

A 75-year-old hypermetropic man presented with right acute angle closure glaucoma of 48 hours' duration. The intraocular pressure was 78 $\mathrm{mmHg}$, the anterior chamber was shallow, and the angle was closed throughout $360^{\circ}$. The left eye had a very narrow, open angle. Treatment with acetazolamide $500 \mathrm{mg}$ intravenously and pilocarpine eyedrops $2 \%$ hourly resulted in a fall in intraocular pressure to $12 \mathrm{mmHg}$, with an open angle throughout $360^{\circ}$. Nd-YAG laser iridotomy was performed three days later. A treatment site $1 \mathrm{~mm}$ from the limbus at 10 o'clock was chosen. The iris was a light grey colour, with no crypts. Three $9.0 \mathrm{~mJ}$ pulses resulted in a small $(200 \times 100 \mu \mathrm{m})$ patent iridotomy. Minimal iris bleeding occurred at the time of treatment, but there were no other complications. One week after treatment the iridotomy was patent, though small. The intraocular pressure was $10 \mathrm{mmHg}$. No further eye medication was prescribed.

Two weeks after treatment the patient complained of blurred vision in the right eye. The intraocular pressure was $68 \mathrm{mmHg}$, and gonioscopy revealed $300^{\circ}$ of angle closure superiorly. Medical treatment with topical pilocarpine $2 \%$ and timolol $0.5 \%$ reduced the intraocular pressure to $13 \mathrm{mmHg}$, with an open angle. The iridotomy was small $(50 \times 100 \mu \mathrm{m})$ but patent. Trabeculectomy with peripheral iridectomy was performed, leading to an intraocular pressure of $10 \mathrm{mmHg}$, and $6 / 5$ visual acuity. 


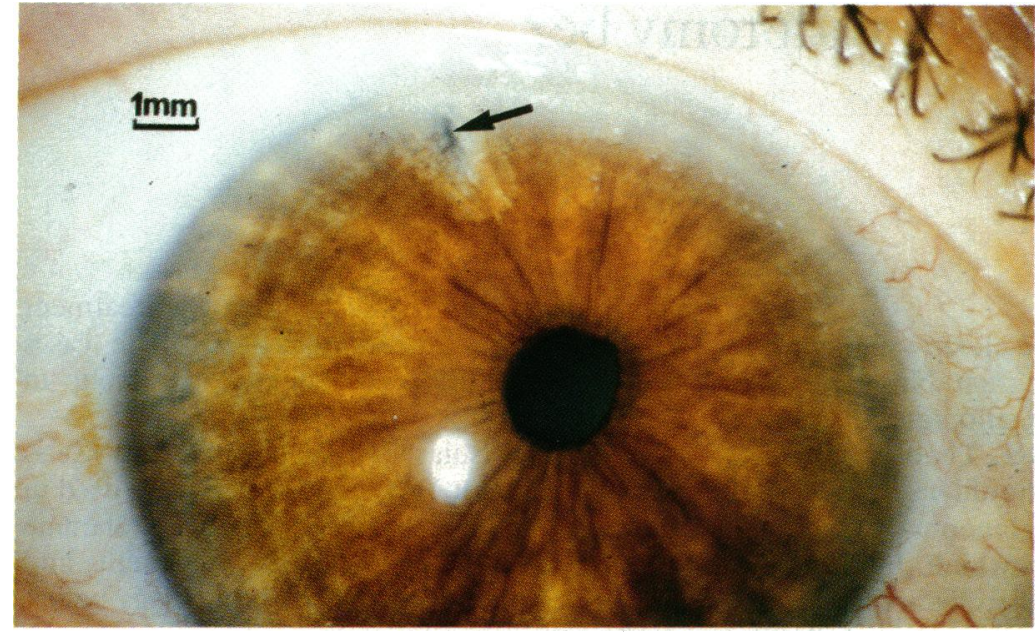

Figure 1 Case 2. Initial iridotomy size too small. Acute angle closure glaucoma developed. Iridotomy diameter $50 \mathrm{\mu m}$. The arrow indicates the very small area of full thickness opening at the centre of the treatment site.

CASE 2

A 65-year-old woman presented with left acute angle closure glaucoma of 24 hours' duration. The intraocular pressure was $58 \mathrm{mmHg}$, the anterior chamber was shallow, and the angle was closed throughout $360^{\circ}$. The right anterior chamber was shallow, with $120^{\circ}$ of angle closure superiorly, and the intraocular pressure was 15 mmHg.

After initial medical control left trabeculectomy was performed.

Further examination of the right eye after treatment with pilocarpine eyedrops $2 \%$ revealed an open angle throughout $360^{\circ}$. Right Nd-YAG laser iridotomy was performed. The treatment site was $1 \mathrm{~mm}$ from the limbus, at 10 o'clock. The iris was brown, with no crypts. Four $9.7 \mathrm{~mJ}$ pulses were used to produce a patent iridotomy, and a further single $9.7 \mathrm{~mJ}$ pulse was used to enlarge the opening.

One week later the iridotomy was patent, though small. The intraocular pressure was 14 $\mathrm{mmHg}$. At review three weeks later the right intraocular pressure was found to be $41 \mathrm{mmHg}$, and the pupil was dilated. Gonioscopy was not

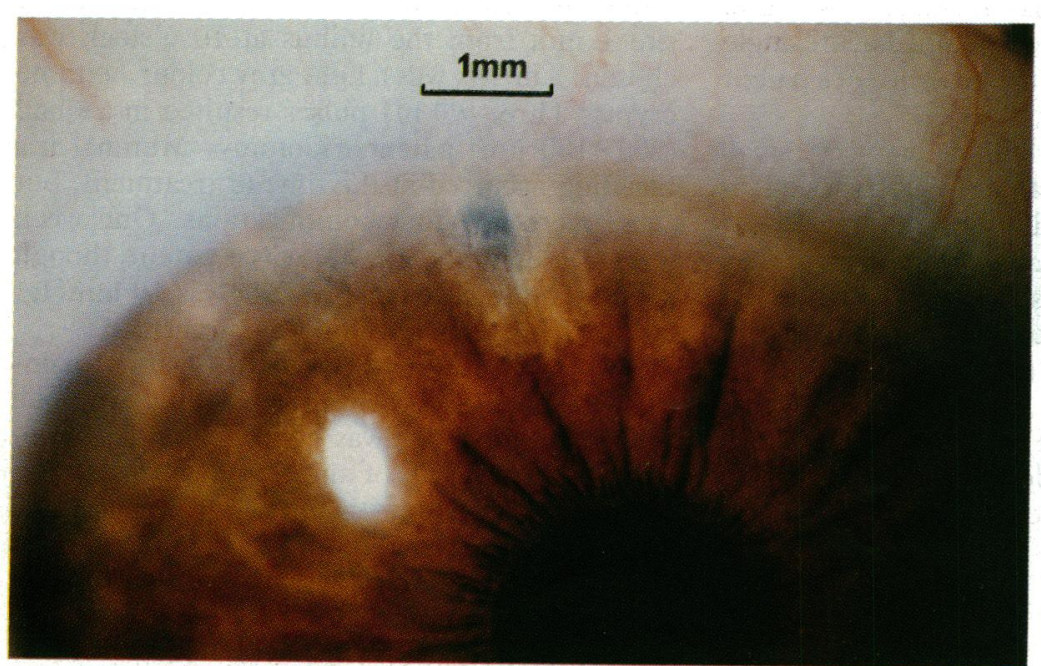

Figure 2 Case 2. Following laser enlargement all symptoms resolved and provocation testing was negative. Iridotomy diameter $200 \mu \mathrm{m}$. performed. The patient had been using cyclopentolate $1 \%$ drops to the left eye, and may have inadvertently contaminated the right eye. After treatment with pilocarpine eyedrops $2 \%$ the intraocular pressure returned to $16 \mathrm{mmHg}$. The iridotomy was patent, but small $-50 \times 50 \mu \mathrm{m}$ (Fig 1). Further laser treatment was given to enlarge the opening. Five $2.0 \mathrm{~mJ}$ Nd-YAG pulses were delivered to the sides of the existing iridotomy, enlarging the opening to $100 \times 100$ $\mu \mathrm{m}$. After this treatment, eyedrops of Betamethasone $0.1 \%$ four times a day and tropicamide $1 \%$ once daily were prescribed. The patient returned four days later complaining of pain and reduced vision in the right eye. The intraocular pressure was $50 \mathrm{mmHg}$, and the pupil was middilated and unreactive. The iridotomy was now situated very peripherally, with iris strands running forwards to the cornea, but appeared to be patent. Gonioscopy was not performed. The intraocular pressure returned to normal after treatment with acetazolamide $500 \mathrm{mg}$ intravenously, and pilocarpine eyedrops $2 \%$ hourly. The iridotomy was patent, but small $(100 \times 100$ $\mu \mathrm{m})$. The angle was open on gonioscopy. Treatment with pilocarpine eyedrops $2 \%$ four times a day was prescribed.

A pilocarpine/phenylephrine provocation test as described by Mapstone ${ }^{18}$ was performed on the right eye one week later and was negative.

Intermittent aching pain above the right eye continued, and further Nd-YAG laser enlargement of the iridotomy was performed six weeks later. Twenty-one $1.1 \mathrm{~mJ}$ pulses were delivered by means of a Wise 103-dioptre contact lens, resulting in some oozing of blood. A further 10 $1.0 \mathrm{~mJ}$ pulses were delivered 30 minutes later. The resulting iridotomy measured $200 \times 200 \mu \mathrm{m}$.

One week later all symptoms had resolved, and the iridotomy measured $100 \times 200 \mu \mathrm{m}$. Pilocarpine/phenylephrine testing was again negative. However, provocation with Tropicamide eyedrops $1 \%$ produced a rise of $9 \mathrm{mmHg}$ in IOP, with folds of iris blocking the trabecular meshwork over $270^{\circ}$ on gonioscopy. At followup three weeks later the intraocular pressure was $17 \mathrm{mmHg}$, with no medication (Fig 2).

CASE 3

An 83-year-old woman presented with intermittent pain in the left eye. The intraocular pressure was $44 \mathrm{mmHg}$, the anterior chamber was deep, and Goldmann gonioscopy revealed $90^{\circ}$ of angle closure superotemporally. The plane of the iris was flat, with an anteriorly placed iris insertion. The right eye had a similar angle configuration, with normal intraocular pressure. Plateau iris configuration was diagnosed. Intraocular pressure in the left eye quickly returned to normal after treatment with acetazolamide $\mathbf{5 0 0}$ mg orally and pilocarpine eyedrops $2 \%$ hourly. Gonioscopy revealed $180^{\circ}$ of angle closure superiorly. Nd-YAG laser iridotomy was performed three days later. The iris was light grey, with no crypts. A treatment site $1 \mathrm{~mm}$ from the limbus at 2 o'clock was selected. Four $10.9 \mathrm{~mJ}$ pulses produced a moderately large $(200 \times 400$ $\mu \mathrm{m})$ patent iridotomy. There was minimal iris bleeding at the time of treatment. 
After this treatment, eyedrops of Timolol $0.5 \%$ twice daily, cyclopentolate $1 \%$ twice daily, and betamethasone $0.1 \%$ four hourly were prescribed. Fourteen days later the patient returned with pain and reduced vision of 24 hours' duration in the left eye. The intraocular pressure was $50 \mathrm{mmHg}$. After treatment with Pilocarpine eyedrops $2 \%$ and timolol eyedrops $0.5 \%$ the intraocular pressure fell to $7 \mathrm{mmHg}$. The iridotomy was patent and moderately large $(200 \times 400 \mu \mathrm{m})$. Identation gonioscopy revealed goniosynechiae over $150^{\circ}$ superiorly, with plateau iris configuration. A sector iridectomy was performed. On further follow-up the intraocular pressure in the left eye was found to be $28 \mathrm{mmHg}$ on no treatment, falling to $21 \mathrm{mmHg}$ on addition of timolol eyedrops $0 \cdot 5 \%$ twice daily. Gonioscopy revealed an open angle throughout $360^{\circ}$.

CASE 4

A 71-year-old hypermetropic woman presented with left acute angle closure glaucoma of eight hours' duration. The intraocular pressure was $46 \mathrm{mmHg}$ and the anterior chamber was shallow. The right eye had a normal intraocular pressure and a shallow anterior chamber. After medical treatment with acetazolamide $500 \mathrm{mg}$ intravenously and pilocarpine eyedrops $2 \%$ hourly the left intraocular pressure fell to $9 \mathrm{mmHg}$ within two hours. Goldmann gonioscopy revealed $150^{\circ}$ of angle closure superiorly. The right eye also had a very narrow angle, with $150^{\circ}$ of angle closure superiorly.

Nd-YAG laser iridotomy was attempted in the left eye the next day. The iris was brown, with no crypts. Three $9.9 \mathrm{~mJ}$ pulses were delivered to two sites, with no penetration. Three days later four $10.2 \mathrm{~mJ}$ pulses were delivered, two to each of the previous treatment sites. The final pulse produced a very small, patent iridotomy at 2 o'clock, in the mid iris position. A single enlarging pulse of $9.2 \mathrm{~mJ}$ was delivered to this site on the following day. A very small $(50 \mu \mathrm{m})$ iridotomy resulted. The patient was discharged home with betamethasone eyedrops $0 \cdot 1 \%$ twice daily and cyclopentolate eyedrops $1 \%$ once daily to the left eye.

She returned six days later complaining of severe pain in the left eye. The intraocular pressure was $45 \mathrm{mmHg}$, and the anterior chamber was very shallow. The iridotomy was patent but very small. The intraocular pressure quickly fell to $8 \mathrm{mmHg}$ after treatment with

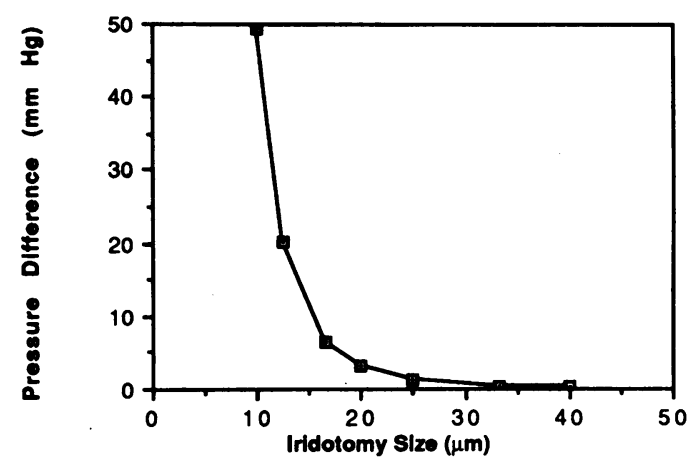

Figure 3 Pressure difference across the iris as a function of iridotomy size. acetazolamide $500 \mathrm{mg}$ intravenously and pilocarpine eyedrops $2 \%$ hourly. A surgical peripheral iridectomy was performed the next day.

Postoperatively the patient was treated with betamethasone eyedrops $0 \cdot 1 \%$ four times daily and tropicamide eyedrops $1 \%$ once daily. Two days later she complained of pain over the left eye. The pupil was dilated, and the peripheral iridectomy was patent. The intraocular pressure was $41 \mathrm{mmHg}$. Gonioscopy was not tolerated. The intraocular pressure fell to $10 \mathrm{mmHg}$ after treatment with acetazolamide $250 \mathrm{mg}$ orally twice daily, pilocarpine eyedrops $2 \%$ four times daily, and timolol eyedrops $0.5 \%$ twice daily. Long term treatment with pilocarpine eyedrops $2 \%$ four hourly and timolol eyedrops $0.5 \%$ twice daily has been necessary to maintain normal intraocular pressure in the left eye.

MATHEMATICAL MODEL

A very small iridotomy may be of inadequate physical size to by-pass pupil block adequately. Given a constant rate of aqueous flow, the pressure difference across an iridotomy increases as the size of the iridotomy decreases. Increased posterior chamber pressure relative to anterior chamber pressure will result in iris bombé. The degree of bombé is dependent on the rigidity of the iris and the pressure difference across the iris. At a critical level, dependent on peripheral anterior chamber depth, iris bombé may become sufficient to cause iridocorneal contact over a large enough extent of the angle to precipitate acute angle closure glaucoma. 16171920

The minimum 'safe' size of an iridotomy may be calculated from a simple mathematical model. Firstly, an assessment is made as to whether aqueous flow is laminar or turbulent through an iridotomy aperture of $10 \mu \mathrm{m}$. On the assumption that aqueous viscosity equals the viscosity of water $\left(\eta=10^{-3}\right.$ Newton seconds per square metre), aqueous density equals the density of water $(\varrho=1000$ kilograms per cubic metre), and aqueous flow, $Q$, is approximately $2 \mu \mathrm{l}$ per minute $\left(3.3 \times 10^{-11}\right.$ cubic metres per second), as measured by Bloom et al, ${ }^{21}$ then Reynolds' number for the system may be calculated.

If the iridotomy size (d) is $10 \mu \mathrm{m}$ diameter, the aqueous velocity

$\mathrm{v}=$ flow $/ \pi \mathrm{r}^{2}=4 \cdot 2 \times 10^{-1} \mathrm{~ms}^{-1}$.

Reynolds' number for the system $R=\varrho v d / \eta, R=42$.

If $R$ is considerably less than 1000 , flow is laminar. Aqueous flow through an iridotomy of diameter $10 \mu \mathrm{m}$ or larger will be laminar.

Secondly, the iridotomy channel may be considered to be a pipe of length $50 \mu \mathrm{m}$ on the assumption that the peripheral iris thickness is $50 \mu \mathrm{m}$. The pressure drop (pd) along a pipe of length $1=50 \mu \mathrm{m}$, diameter $\mathrm{d}=100 \mu \mathrm{m}$, at flow rate $Q$ is expressed by the formula:

$$
\begin{aligned}
\mathrm{pd} & =128 \eta 1 \mathrm{Q} / \pi \mathrm{d}^{4} \\
& =128 \times 10^{-3} \times 5 \times 10^{-5} \times 3.3 \times 10^{-11} / 3 \cdot 142 \times \\
& 10^{-16} \\
& =0.672 \mathrm{Nm}^{-2} \\
& =0.00494 \mathrm{mmHg}, \text { as } 1 \mathrm{mmHg}=136 \mathrm{Nm}^{-2} .
\end{aligned}
$$

An iridotomy of $100 \mu \mathrm{m}$ diameter will have a pressure drop of $0.00494 \mathrm{mmHg}$ across it on the assumption of total pupil block. 
However, as pd is proportional to $1 / \mathrm{d}^{4}$, if the diameter of the iridotomy were $50 \mu \mathrm{m}$ pd would be $0.079 \mathrm{mmHg}$, under the same conditions. If the iridotomy were $25 \mu \mathrm{m}$, the pd would be 1.27 $\mathrm{mmHg}$. If the iridotomy were $12.5 \mu \mathrm{m}$, the pd would be $20.2 \mathrm{mmHg}$, and if the iridotomy were $10 \mu \mathrm{m}$ the pd would be $49.4 \mathrm{mmHg}$. It is not difficult to conceive that a pressure difference of $10-20 \mathrm{mmHg}$ across the iris could result in significant iris bombé, with iridocorneal contact and angle closure. Such a pressure difference could develop if the size of the iridotomy was between 10 and $15 \mu \mathrm{m}$ in diameter (Fig 3).

\section{Discussion}

Curran observed in 1920 that in some patients with glaucoma the passage of aqueous through the pupil appeared to be impeded by the iris 'hugging the lens over too great a surface extent'. ${ }^{2}$ Surgical iridotomy allowed free drainage of fluid from the posterior chamber to the anterior chamber, with posterior movement of the iris and a reduction in intraocular pressure. Barkan elaborated the concept of pupil block as the causative mechanism of iris bombé, with resulting angle closure. ${ }^{22}$ A peripheral iridectomy causes 'collapse' of iris bombé, resulting in widening of the 'entrance' to the angle. ${ }^{22}$ Peripheral anterior chamber depth, but not central anterior chamber depth, increases following peripheral iridectomy ${ }^{172324}$ owing to resolution of iris bombé.

Failure of surgical peripheral iridectomy, or laser iridotomy, as evidenced by development of acute angle closure glaucoma, may occur by one of two mechanisms. A small number of cases of angle closure glaucoma are related to 'plateau iris configuration' - an anteriorly placed iris root, flat iris plane, and a normal or near normal anterior chamber depth. Pupil dilatation results in peripheral iris bunching, with occlusion of the trabecular meshwork. ${ }^{1325}$ As pupil block is not causative, surgical peripheral iridectomy or laser iridotomy are relatively ineffective in preventing further attacks of acute angle closure glaucoma. One of our patients (case 3) probably had plateau iris syndrome, and developed acute angle closure glaucoma following mydriasis despite the presence of a relatively large, patent iridotomy.

Case 4 developed acute angle closure glaucoma following mydriasis despite the presence of a small patent iridotomy and subsequently a larger surgical iridectomy. The mechanism of acute intraocular pressure rise may have been iris crowding in the angle. Plateau iris configuration was not present. Perhaps the term 'angle crowding' angle closure glaucoma would be a more appropriate term to use for eyes which develop acute angle closure glaucoma following mydriasis in the presence of a patent peripheral iridectomy. The cases of two other patients with moderately large peripheral iridectomies who developed acute angle closure glaucoma while using mydriatic drops have been reported. ${ }^{1+15}$

Mydriatic or dark room provocation tests following peripheral iridectomy have been found to be positive in $3-19 \%$ of cases. ${ }^{25-28}$ Gonioscopy at the end of a positive test in eyes with patent peripheral iridectomies has revealed angle closure in some cases, due to angle crowding. ${ }^{25} 29$ However, in other eyes the angle has been open. ${ }^{25-27}$ Raised intraocular pressure in these eyes may be explained by the outflow facility lowering effect of anticholinergic drugs. ${ }^{30}$ Surgical peripheral iridectomy appears entirely to overcome pupil block. No cases of acute angle closure glaucoma due to iris bombé have been reported in the presence of a patent surgical iridectomy.

Mydriatic provocation tests following argon laser iridotomy ${ }^{3132}$ and Nd-YAG laser iridotomy ${ }^{33}$ have given similar results to those performed following surgical peripheral iridectomy. However, pilocarpine/phenylephrine tests have been negative in all eyes tested following argon laser iridotomy ${ }^{34}$ or $\mathrm{Nd}$ YAG laser iridotomy. ${ }^{812}$ The test produces only moderate mydriasis, and increased tone in both the sphincter and dilator muscles of the iris may prevent angle crowding. The test induces maximal pupil block, ${ }^{35}$ and failure to obtain any positive results suggests that pupil block was fully overcome in all cases tested.

Acute angle closure glaucoma in the presence of a small, patent argon laser iridotomy has previously been reported in two patients. ${ }^{910}$ Mandelkorn et al reported a case of acute angle closure glaucoma following a $50 \mu \mathrm{m}$ diameter argon laser iridotomy. ${ }^{9}$ Further argon laser treatment was applied to the site, resulting in satisfactory long term pressure control. Brainard reported a similar case, in an eye with a $75 \mu \mathrm{m}$ diameter argon laser iridotomy. ${ }^{10}$ Further argon laser treatment to enlarge the iridotomy to $125 \mu \mathrm{m}$ resulted in satisfactory long term intraocular pressure control, suggesting inadequate iridotomy size was responsible for initial failure.

Acute angle closure glaucoma has also been reported in the presence of a small, but patent Nd-YAG laser iridotomy. ${ }^{11} 1233$ Brazier reported two cases. One was treated by surgical peripheral iridectomy, with no further complications. The second was treated with miotics." Wishart and Hitchings reported two cases of acute angle closure glaucoma and two cases of subacute angle closure glaucoma in the presence of patent Nd-YAG laser iridotomies. ${ }^{33}$ In three cases the iridotomy was $100 \mu \mathrm{m}$ diameter and in the fourth $150 \mu \mathrm{m}$. Gray et al reported two cases of acute angle closure glaucoma in the presence of a small, patent iridotomy..$^{12}$ One developed acute angle closure glaucoma while using pilocarpine eye drops $4 \%$, and was treated by trabeculectomy. The second was treated by a further iridotomy. Intraocular pressure subsequently remained normal in both cases. Two of our cases (cases 1 and 2) behaved in a very similar way to the two cases reported by Gray et al. Case 1 responded to trabeculectomy, and case 2 responded to laser enlgargement of the iridotomy. The occurrence of acute angle closure glaucoma in the presence of a small, but patent, iridotomy which responds to surgical peripheral iridectomy or laser enlargement, suggests the size of the initial iridotomy was inadequate.

Wishart studied iris bombé using slit-lamp photographic assessment of peripheral anterior chamber depth. In four eyes with very small iridotomies and progressive narrowing of the 
angle, repeat laser iridotomy resulted in deepening of the peripheral anterior chamber. ${ }^{33}$ This study provides further evidence that very small iridotomies may not fully relieve iris bombé.

We have calculated that the minimum functional iridotomy size should be approximately 15 $\mu \mathrm{m}$, as previously suggested by Wheeler. ${ }^{36}$ However, our clinical experience, and that of others, ${ }^{91233}$ shows that iridotomies in the range of 50-150 $\mu \mathrm{m}$ diameter may fail to prevent acute angle closure glaucoma.

Several factors may contribute to the reduction in size of an iridotomy of theoretically 'safe' size below that needed to prevent acute angle closure glaucoma. Most cases of acute angle closure glaucoma. following Nd-YAG laser iridotomy have occurred within one month of treatment. Naveh et al observed rapid diminution of Nd-YAG laser iridotomy size within an hour of treatment in six eyes. ${ }^{37}$ In each case a return to original treatment size occurred within two weeks. Localised iris oedema round the treatment site could be responsible.

Re-closure of Nd-YAG laser iridotomies due to iris pigment epithelium proliferation is unusual, and tends to occur 1-4 months after treatment. ${ }^{11} 12333738$ Re-closure resulted in acute angle closure glaucoma in two eyes reported on by Wishart and Hitchings ${ }^{33}$ and one eye reported on by Gray et al. ${ }^{12}$

Case 2 developed acute angle closure glaucoma following mydriasis. Pupil dilatation might reduce the surface area of some iridotomies, and folds of iris tissue might functionally block an iridotomy when the pupil is dilated. Mydriatics might therefore produce acute angle closure glaucoma in the presence of a patent iridotomy by two mechanisms - pupil block due to reduction of iridotomy size, and angle crowding.

To answer the question, How large must an iridotomy be to prevent acute angle closure glaucoma? several factors must be considered. In forms of angle crowding angle closure glaucoma, such as plateau iris syndrome, a large iridotomy will not prevent the development of acute angle closure glaucoma following mydriasis, as pupil block is not causative.

Theoretical modelling suggests an iridotomy of 10-15 $\mu \mathrm{m}$ diameter will prevent angle closure glaucoma due to pupil block. However, an iridotomy of greater size may fall below this critical level due to early post-treatment oedema, late pigment epithelium proliferation, or pupil dilatation. Wishart and Hitchings reported subacute angle closure glaucoma in an eye with a 150 $\mu \mathrm{m}$ diameter iridotomy. ${ }^{33}$ All other cases of acute angle closure glaucoma in the presence of a patent iridotomy have occurred in eyes with an iridotomy of less than $100 \mu \mathrm{m}$ in diameter. The smallest potentially safe size of an iridotomy would therefore appear to be $100 \mu \mathrm{m}$. However, in order to incorporate a safety margin, we would recommend that iridotomies should be at least $150-200 \mu \mathrm{m}$ in diameter.

Provocation tests have not been helpful in identifying eyes at risk, as eyes developing acute angle closure glaucoma following Nd-YAG laser iridotomy have done so before a provocation test had been performed. ${ }^{812} 33$ If provocation tests are to be employed, we suggest the use of tropi- camide eyedrops $1 \%$ soon after the iridotomy has been performed, in order to detect cases at risk of developing acute angle closure glaucoma. Routine pupil dilatation to prevent posterior synechiae formation following laser iridotomy may be more safely achieved with phenylephrine eyedrops $10 \%$, with measurement of intraocular pressure following dilatation. Lowe has shown that phenylephrine very rarely results in a rise in intraocular pressure following peripheral iridectomy, ${ }^{15}$ and the effect may be quickly reversed using thymoxamine eyedrops $0.5 \%$ if a pressure rise does occur.

Dr N A Fleck, Department of Engineering, University of Cambridge, developed the mathematical model. Professor C Phillips and Dr J F Cullen, Department of Ophthalmology, Edinburgh Royal Infirmary, made helpful comments during preparation of the manuscript. Mr Stuart Gairns, ophthalmic photographer, assisted in preparation of the illustrations.

This paper forms part of an ongoing research programme funded by the W H Ross Foundation.

1 Von Graefe A. Über die Iridectomie bei Glaucoma und über den glaucomatosen Process. Graefes Arch Clin Exp Ophthalmol 1857; 3: 456-555.

2 Curran EJ. A new operation for glaucoma involving a new principle in the aetiology and treatment of chronic primary principle in the aetiology and treatment of ch
glaucoma. Arch Ophthalmol 1920; 49: 131-55.

3 Curran EJ. Peripheral iridotomy in acute and chronic glaucoma: some results after 10 years duration. Anatomica glaucoma: some results after 10 years duration. Anatomical 51: $520-38$

4 Gifford H. Peripheral iridotomy (Curran) in the treatment of glaucoma. Am f Ophthalmol 1921; 4: 889-94

5 Chandler PA. Narrow angle glaucoma. Arch Ophthalmol 1952 47: 695-716.

6 Abraham RK, Miller GL. Outpatient argon laser iridectomy for angle closure glaucoma: a two year study. Ophthalmolog 1975; 79: OP529-38.

7 Frankhauser F, Roussel P, Steffen J, Van Der Zyphen E, Chrenkova A. Clinical studies on the efficiency of high power laser radiation upon some structures of the anterio segment of the eye. First experience of the treatment of some pathological conditions of the anterior segment of the human eye by means of a Q-switched laser system. Int Ophthalmol 1981; 3: 129-39.

8 Fleck BW, Fairley A, McGlynn C. A prospective comparison of Nd:YAG laser iridotomy and surgical peripheral of $\mathrm{Nd}$ iridectomy in the treatment of acute angle closure glaucoma. iridectomy in the treatment of acute angle closure glaucoma.
Proceedings of the XXVI International Congress of OphthalProceedings of the XXVI Intern
mology. Singapore (in press).

9 Mandelkorn R, Mendelsohn A, Olander $\mathrm{K}$, et al. Short exposure times in argon laser iridotomy. Ophthalmic Sur $1981 ; 12: 805-9$.

10 Brainard JO, Landers JH, Shock JP. Recurrent angle closure glaucoma following a patent 75-micron laser iridotomy: case report Ophthalmic Surg 1982; 13: 1030-2.

11 Brazier DJ. Neodymium-YAG laser iridotomy. $7 R$ Soc $M e$ 1989; 79: 658-60

12 Gray RH, Hoare Nairn J, Ayliffe WHR. Efficacy of Nd-YAG laser iridotomies in acute angle-closure glaucoma. $\mathrm{Br}$ Ophthalmol 1989; 73: 182-5.

13 Wand M, Grant WM, Simmons RJ, Hutchinson BT. Plateau iris syndrome. Ophthalmology 1977; 83: 122-30.

14 Douglas WHG, Strachan IM. Surgical safety of prophylactic peripheral iridectomy. Brf Ophthalmol 1967; 51: 459-62.

15 Lowe RF. Primary angle-closure glaucoma: postoperative acute glaucoma after phenylephedrine eyedrops. Am Ophthalmol 1968; 65: 552-4.

16 Phillips CI. Sectorial distribution of goniosynechiae. $B$ 7 Ophthalmol 1956; 40: 129-35.

17 Phillips CI. Closed-angle glaucoma. Significance of sectorial variations in angle depth. Br $\mathcal{F}$ Ophthalmol 1956; 40: 136-43.

18 Mapstone R. Provocative tests in closed-angle glaucoma. $\mathrm{Br}$ f Ophthalmol 1976; 60: 115-9.

19 Bhargava SK, Leighton DA, Philips CI. Early angle-closure glaucoma. Distribution of iridotrabecular contact and response to pilocarpine. Arch Ophthalmol 1973; 89: 369-72.

20 Mapstone R. One gonioscopic fallacy. Br $\mathcal{O}$ Ophthalmol 1979 63: $221-4$.

21 Bloom JN, Levene RZ, Thomas G, Kimura R. Fluorophotometry and the rate of aqueous flow in man. Arch Ophthalmol 1976; 94: 435-43.

22 Barkan $O$. Iridectomy in narrow angle glaucoma. $A$ $\mathcal{F}$ Ophthalmol 1954; 37: 504-19.

23 Jacobs IH, Krohn DL. Central anterior chamber depth afte laser iridectomy. Am f Ophthalmol 1980; 89: 865-7.

24 Lee DA, Brubaker RF, Ilstrup DM. Anterior chamber dimensions in patients with narrow angles and angle-closure glaucoma. Arch Ophthalmol 1984; 102: 46-50.

25 Lowe RF. Primary angle closure glaucoma - investigation after surgery for pupil block. Am $\mathcal{F}$ Ophthalmol 1964; 57 931-8.

26 Harris LS, Galin MA. Cycloplegic provocative testing. Arch Ophthalmol 1969; 81 : 356-8. 
27 Friedman Z, Neumann E. Comparison of prone position, dark room, and mydriatic tests for angle closure glaucoma before and after peripheral iridectomy. Am $\mathcal{F}$ Ophthalmol 1972; 74: $24-7$.

28 Hung PT, Chou LH. Provocation and mechanism of angleclosure glaucoma after iridectomy. Arch Ophthalmol 1979,

29 Godel V, Stein R, Feiler-Ofrey V. Angle-closure glaucoma following peripheral iridectomy and mydriasis. $A m$ 30 Oph

.

31 Karmon G. Vender T, Savir H. Evaluation of laser iridectomy in angle-closure glaucoma: provocative tests. $\mathrm{Br} \mathcal{J ~ O p h}$

32 Yassur Y, Melamed S, Cohen S, Ben-Sira I. Laser iridotomy in closed angle glaucoma. Arch Ophthalmol 1979; 97: 1920-1.

3 Wishart PK, Hitchings RA. Neodymium YAG and dye laser iridotomy - a comparative study. Trans Ophthalmol Soc UK 1986; 105: $521-40$.

34 Harrad RA, Stannard KP, Shilling JS. Argon Laser iridotomy. Br f Ophthalmol 1985; 69: 368-72. •

35 Mapstone R. Mechanics of pupil block. Br 7 Ophthalmol 1968 52: 19-25.

heeler CB. Laser iridectomy. Phys Med Biol 1977; 6:

7 Naveh N, Zborowsky-Gutman L, Blumenthal M. Neodymium-YAG laser iridotomy in angle closure glaucoma

38 McAllister JA, Schwartz LW, Moster M, Spaeth GL. Laser peripheral iridectomy comparing Q-switched neodymium
YAG with argon. Trans Ophthalmol Soc UK 1985; 104: 67-9. 